\title{
Metabolomics of Thrips Resistance in Pepper (Capsicum spp.) Reveals Monomer and Dimer Acyclic Diterpene Glycosides as Potential Chemical Defenses
}

\author{
Mirka Macel $^{1}$ (D) - Isabella G. S. Visschers ${ }^{1} \cdot J_{a n n y}$ L. Peters ${ }^{2} \cdot$ Iris F. Kappers ${ }^{3} \cdot$ Ric C. H. de Vos ${ }^{4} \cdot$ Nicole M. van Dam $^{1,5,6}$
}

Received: 17 January 2019 /Revised: 9 May 2019/Accepted: 13 May 2019 / Published online: 8 June 2019

(C) The Author(s) 2019

\begin{abstract}
The development of pesticide resistance in insects and recent bans on pesticides call for the identification of natural sources of resistance in crops. Here, we used natural variation in pepper (Capsicum spp.) resistance combined with an untargeted metabolomics approach to detect secondary metabolites related to thrips (Frankliniella occidentalis) resistance. Using leaf disc choice assays, we tested 11 Capsicum accessions of $C$. annuum and $C$. chinense in both vegetative and flowering stages for thrips resistance. Metabolites in the leaves of these 11 accessions were analyzed using LC-MS based untargeted metabolomics. The choice assays showed significant differences among the accessions in thrips feeding damage. The level of resistance depended on plant developmental stage. Metabolomics analyses showed differences in metabolomes among the Capsicum species and plant developmental stages. Moreover, metabolomic profiles of resistant and susceptible accessions differed. Monomer and dimer acyclic diterpene glycosides (capsianosides) were pinpointed as metabolites that were related to thrips resistance. Sucrose and malonylated flavone glycosides were related to susceptibility. To our knowledge, this is the first time that dimer capsianosides of pepper have been linked to insect resistance. Our results show the potential of untargeted metabolomics as a tool for discovering metabolites that are important in plant - insect interactions.
\end{abstract}

Keywords Capsianosides $\cdot$ Frankliniella occidentalis $\cdot$ Liquid chromatography-mass spectrometry $\cdot$ Insects $\cdot$ Solanaceae $\cdot$ Thrips

Electronic supplementary material The online version of this article (https://doi.org/10.1007/s10886-019-01074-4) contains supplementary material, which is available to authorized users.

Mirka Macel

mirkamacel@gmail.com

1 Molecular Interaction Ecology, Institute of Water and Wetland Research (IWWR), Radboud University, P.O. Box 9010, 6500 GL Nijmegen, The Netherlands

2 Molecular Plant Physiology, Institute of Water and Wetland Research (IWWR), Radboud University, P. O. Box 9010, 6500

GL Nijmegen, The Netherlands

3 Laboratory of Plant Physiology, Wageningen University and Research, P.O. Box 658, 6700 AR Wageningen, The Netherlands

4 Wageningen Plant Research, Bioscience, Wageningen University and Research, P.O. Box 16, 6700 AA Wageningen, The Netherlands

5 German Centre for Integrative Biodiversity Research (iDiv) Halle-Jena-Leipzig, Deutscher Platz 5e, 04103 Leipzig, Germany

6 Institute of Biodiversity, Friedrich Schiller University Jena, Dornburger-Str. 159, 07743 Jena, Germany

\section{Introduction}

Plant resistance to insects is often reduced in crops compared to their wild ancestors, due to breeding efforts to minimize the unwanted taste or toxic effects of some of the plants' natural chemical defenses. Modern varieties of cabbage (Brassica oleraceae) for example, have very low glucosinolate levels, typical anti-herbivore defenses in the Brassicaceae family (Gols et al. 2008). To grow healthy crops, application of insecticides has therefore become a necessity. However, not all insects can be controlled effectively by insecticides, partly due to a recent increase in insecticide resistant insect populations (Bass and Jones 2018). Moreover, sustainable agricultural practices call for finding natural insect resistance in crops to reduce the need for insecticides that are harmful to the environment, e.g. neonicotinoids (Hallmann et al. 2014). Because genetically modified crops are largely banned from Europe, novel sources of resistance preferably come from wild crop relatives that can be used in breeding schemes. 
Capsicum species (Solanaceae) are grown world-wide as economically important crops, such as hot and sweet peppers, which are mostly Capsicum annuum L. and Capsicum chinense L.. A major pest on Capsicum are thrips, an order of tiny insects that can cause severe damage (Steenbergen et al. 2018). In greenhouses in Western Europe, western flower thrips (Frankliniella occidentalis) is the prevalent pest species (Kirk and Terry 2003). F. occidentalis is notoriously difficult to control with insecticides, due to its thigmotactic behavior and fast evolving insecticide resistance (Bielza 2008). There are a few studies on insect resistance in Capsicum (e.g. Fery and Schalk 1991; Maharijaya et al. 2011, 2012; Mollema and Cole 1996; Yang et al. 2011), but the mechanisms underlying insect resistance in this genus still remain largely unknown. Next to physical defenses such as hairs, almost all plants produce chemical defenses. In this study we aim to identify the chemical defenses of Capsicum species by using the natural variation in thrips resistance among Capsicum accessions.

Plants produce a wealth of primary and secondary metabolites. Plant chemical defenses are mostly secondary metabolites (Schoonhoven et al. 2005). The estimated number of secondary metabolites in the plant kingdom is 200.000 (Weckwerth 2003). Plant secondary chemistry is often highly species specific, with some metabolites only occurring in a single species or genus (Macel et al. 2014; Schweiger et al. 2014). Studies on plant chemical defenses have mostly focused on well-known plant secondary metabolites such as alkaloids or glucosinolates. However, these known chemical defenses are only a fraction of all plant secondary metabolites. Hence, bioactive metabolites may be overlooked when using targeted analytical approaches. Novel analytical techniques and advanced bioinformatics make it possible to detect and identify an unprecedented number of metabolites. Using untargeted metabolomics analyses, many metabolites of different compound classes can be detected in a single analysis (Macel et al. 2010). The benefit is that next to the 'knowns' also yet unknown metabolites can be detected, which are related to plant resistance to herbivores or pathogens (Leiss et al. 2009). The downside of untargeted metabolomics is that some of the unknowns will remain unknown because annotation of the detected metabolites is still a daunting task (Peters et al. 2018).

Here, we used an untargeted metabolomics screening approach in combination with thrips feeding assays to understand the mechanisms of insect resistance in Capsicum. The chemistry of Capsicum fruits is well-studied, mostly in relation to food quality and flavor (Kim et al. 2017; Martín et al. 2017; Wahyuni et al. 2014). Capsaicinoids in the fruits, alkaloids that give the hot peppers their pungent taste, are known to have anti-microbial activity (Tewksbury et al. 2008). Chemistry the leaves is less well investigated, but several studies indicate that secondary plant metabolites of Capsicum play a role in pathogen and insect resistance. For example, the terpenoid capsidiol plays a role in induced defenses against pathogens (Lee et al. 2017). Volatiles emitted by $C$. annuum plants can attract the Chilli thrips Scirtothrips dorsalis (Shivaramu et al. 2017). High levels of tocopherols and low levels of aromatic amino acids in leaves of Capsicum have been related to thrips resistance (Maharijaya et al. 2012; Mollema and Cole 1996). In addition, a recent study on metabolite QTLs and thrips resistance using a C. annuum $\mathrm{x}$ $C$. chinense F2 mapping population showed that high levels some acyclic diterpene glycosides were weakly but significantly correlated to thrips mortality (Maharijaya et al. 2018).

We selected 11 Capsicum accessions from a group of 40 accessions that were screened for resistance to the western flower thrips F. occidentalis (Visschers et al. 2019). These 11 accessions, seven $C$. annuum and four $C$. chinense, were classified either resistant or susceptible to $F$. occidentalis. In this study, the same 11 accessions were tested again for thrips resistance using leaf disc feeding choice-assays. Because insect resistance may be plant age dependent (Barton and Koricheva 2010; Cipollini and Redman 1999; Visschers et al. 2019), we tested leaves from plants in the vegetative stage as well as leaves from flowering plants in the feeding choice assays. Leaves of these plants were also analyzed with untargeted LC-MS metabolomics. Multivariate analyses were used to link the metabolomic profiles with the thrips feeding damage in order to pinpoint metabolites related to thrips resistance or susceptibility in Capsicum.

\section{Material and Methods}

\section{Capsicum Accessions and Plant Growth Conditions}

Eleven Capsicum accessions were selected from a thrips resistance screening of 40 accessions. These eleven accessions were selected for being either most or least resistant (Visschers et al. 2019). Original seed material was provided by the Center for Genetic Resources (CGN) of Wageningen University and Research Centre, The Netherlands (http:// cgngenis.wur.nl/) (Table S1). Seeds were multiplied by selfing three plants per accession in the greenhouse, which were combined in a bulk seed lot per accession. F1 seeds were germinated on sterile glass beads with demi water in a growth cabinet (Snijders DeLuxe, Tilburg, The Netherlands) at $25 / 20{ }^{\circ} \mathrm{C}, 16 / 8 \mathrm{~L} / \mathrm{D}, 70 \%$ humidity. F1 seeds were germinated in closed plastic cups $(7 \mathrm{~cm}$ diameter) on sterile glass beats (1 mm diameter) and demi water in a growth cabinet at $30 / 20{ }^{\circ} \mathrm{C}, 16 / 8 \mathrm{~L} / \mathrm{D}, 70 \%$ humidity. When the first two true leaves had developed, the seedlings were transplanted to plastic pots $(11 \mathrm{~cm} \times 11 \mathrm{~cm} \times 12 \mathrm{~cm})$ containing commercial potting soil. The pots were placed in a greenhouse, inside an insect-free net cage at 16/8 L/D and minimum temperatures set to $20^{\circ} \mathrm{C} / 17^{\circ} \mathrm{C}$ (day/night). Natural light was supplemented with Greenpower lights. Plant were 
given nutrient solution once a week (1.8\% Kristalon Label Blue, Yara, Grimsby, UK). For each accession 8 to 16 plants were grown and included in the metabolomics analysis. A subset of the plants ( 8 to 10 ) of each accession were also used in the thrips choice assays.

\section{Thrips Choice Leaf Disc Assay}

After 4 weeks of growth, when all plants were still in the vegetative stage, one fully grown leaf from the upper part per plant was used for thrips feeding assessment. Per accession 8-10 plants were used for this choice experiment. Leaf disc experiments were performed as described by Visschers et al. (2018a). Briefly, using a cork borer, two leaf discs $(1.5 \mathrm{~cm}$ diameter) were punched from each leaf, thereby avoiding the mid-vein. A leaf disc from each accession was placed on a drop of $1.5 \%$ slightly liquid agar with the abaxial side up in a Petri dish ( $9 \mathrm{~cm}$ diameter). Each Petri dish $(n=20)$ thus contained 11 leaf discs (placed in a circle), each representing 1 of the 11 accessions. The order of the leaf discs was randomized for each replicate, the first disc of each set was indicated with a star underneath the Petri dish. Ten Petri dishes were inoculated with thrips, the other ten were used as controls. Per inoculated Petri dish, $22 \mathrm{~L} 1 / \mathrm{L} 2$ F. occidentalis larvae, reared on green beans and starved for $24 \mathrm{~h}$ prior to experiments, were placed in the middle of the dish using a small painting brush. All Petri dishes were sealed with Parafilm and placed in the same climate cabinet as used for insect rearing. Petri dishes without thrips were directly sealed with Parafilm and used for correction during image analysis. After $48 \mathrm{~h}$ thrips were removed, and thrips damage was assessed using imaging software (Visschers et al. 2018b). After 17 weeks of plant growth, when all plants were flowering, the thrips leaf disc choice assay was repeated, again using leaves from the upper part of the plant.

\section{LC-MS Sample Preparation and Metabolite Extraction}

At the same time when leaves were harvested for the choice assay, one leaf of the same age per plant was harvested for the untargeted LC-MS based metabolomics profiling. Per accession, 8-16 individual plants were used for the chemical analyses. Directly upon harvesting, leaves were flash frozen in liquid nitrogen, freeze dried and stored at $-80^{\circ} \mathrm{C}$. Freeze dried material was finely ground with a Ball Mill (Retsch MM 300) and $20 \mathrm{mg}$ per sample was used for metabolite extraction for LC-MS. A pool sample was made by combining equal amounts of freeze dried material from all accessions and both developmental stages; this pooled sample was treated similarly and simultaneously as the experimental samples and used as a quality control. Samples were extracted with $1 \mathrm{ml} 75 \%$ methanol $+0.1 \%$ formic acid, sonicated at room temperature at $40 \mathrm{kHz}$ for $15 \mathrm{~min}$ and then centrifuged for $10 \mathrm{~min}$ at
$15000 \mathrm{rpm}$ (De Vos et al. 2007). A $500 \mu \mathrm{l}$ aliquot of each sample was transferred into a 96-well LC-MS plate.

\section{LC-MS(MS) Analyses}

Samples were put in randomized block order for LC-MS analyses. Every 20 samples an extract from the sample pool was placed as a quality control. LC-MS analyses were performed as described previously (Mokochinski et al. 2018). In short, separation of compounds in the crude extracts was performed using an HPLC system (Waters Acquity, Milford, USA) generating a flow rate of $0.19 \mathrm{~mL} \cdot \mathrm{min}^{-1}$ and a $45 \mathrm{~min}$ gradient of 5 to $75 \%$ acetonitrile in water, acidified with $0.1 \%$ formic acid, on a $\mathrm{C}_{18}$ reversed phase column (Luna $150 \times 2 \mathrm{~mm}$ i.d., $3 \mu \mathrm{m}$; Phenomenex, Torrance, USA) kept at $40{ }^{\circ} \mathrm{C}$. Detection of eluting compounds was by a PDA detector (Waters) at 210-600 nm and subsequently an LTQ-Orbitrap FTMS hybrid mass spectrometer (Thermo Scientific, Bremen, Germany). Samples were analyzed in both positive and negative ionization modes. A mass resolution of 60,000 FWHM was employed during data acquisition in a mass range of $\mathrm{m} / \mathrm{z}$ 90-1350. Additional LC-MS/MS analyses on selected samples were performed (van der Hooft et al. 2012).

\section{LC-MS - Data Preprocessing}

Baseline correction and peak-wise alignment was done using Metalign (Lommen 2009). The threshold for signal to noise ratio was set at 3 . Data were filtered by removing peaks that were present in less than 8 samples over the entire dataset. For further multivariate data analyses, the peak amplitudes were normalized to 10,000 total peak amplitude for each sample (relative abundance of peaks compared to the total peak amplitude).

\section{LC-MS Mass Feature Clustering}

Cluster analyses were run in MSClust to group those mass peaks that likely belong to the same metabolite, based on their corresponding retention times and relative abundance across samples (Tikunov et al. 2012). This procedure was repeated with remaining mass peaks not assigned to a cluster in the first cluster analysis, after which the two cluster analyses were combined. The cluster analyses of the negative mode data resulted in 1966 clusters, or so-called reconstructed metabolites, to which roughly half of the peaks could be assigned. The mass clustering was used to identify parent ions and facilitate annotation of the metabolites. The relative abundance of metabolites was expressed in MIC values (i.e. Measured Ion Counts) which was used as parameter for the multivariate analyses. 


\section{Metabolite Annotation}

Metabolite annotation was based on manually checking the putative molecular ion within the clustered mass signals of selected metabolites. Metabolites were then annotated using an in-house database (Wageningen Plant Research Bioscience, the Netherlands) based on comparisons of retention time, accurate mass, isotopic composition, UV spectra and MS/ MS information, as well as on-line available metabolite databases such as KNApSAcK, KEGG and MassBank. Putative acyclic diterpene glycosides detected in negative mode were confirmed by MS analysis in positive mode based on the presence of their common fragment with specific accurate $\mathrm{m} / \mathrm{z}$ 271.2424 (Heiling et al. 2016). In our untargeted metabolomics analyses, we detected double charged fragments that were correlated with thrips resistance. These fragments likely belonged to diterpene glycosides, dimer capsianosides, with a molecular weight higher than our initial $\mathrm{m} / \mathrm{z}$ range. For confirmation of the dimer capsianosides, the LC-MS/MS mass range was extended to $m / z, 150-2000$ and selected samples of accession RU 63 were analyzed in both positive and negative ionization modes. Dimeric capsianosides have an $m / z>1400$, monomers have an $m / z<1400$ (KNApSAcK).

\section{Statistical Data Analyses}

Data analyses were performed in R 3.5.0 (www.r-project.org) and Simca 15 (Umetrics, Umea, Sweden). Thrips choice assay data were calculated as $\mathrm{mm}^{2}$ eaten per leaf disc / total amount of $\mathrm{mm}^{2}$ eaten per petri dish $\times 100$ (relative amount eaten per leaf disc in a petri dish) for each leaf disc. These data were analyzed with a Friedman ANOVA for dependent data. Posthoc pairwise differences in thrips damage between accessions were analyzed with paired Wilcoxon signed ranks tests. Based on the choice assays results, accessions were classified as either resistant (<9\% damage; if no preference among 11 choices, $9 \%$ damage is expected on each disc) or susceptible (>9\% damage). This resistance classification was used in the multivariate analyses.

To investigate the overall chemical diversity of the accessions, the total number of detected mass signals among the accessions and developmental stages were analyzed with a two factorial ANOVA with accession and developmental stage as fixed factors. Within each developmental stage, the difference in detected mass signals between susceptible and resistant accessions was also analyzed with ANOVA, with resistance grouping as fixed factor and accession nested in resistance grouping.

To investigate differences in metabolomic profiles, both the mass signal dataset as well as the cluster dataset (MIC) were used for multivariate analyses and subsequent relevant metabolite selection (peak picking). The mass clustering software is still under development, by using both datasets we achieved the most complete and reliable screening of metabolites. Focus of the data analyses was on the negative MS ionisation mode, which was more consistent in peak detection over time, whereas the positive ionization mode data were mostly used for the annotation of the metabolites. Metabolomic profiles were first explored with unbiased Principal Component Analyses (PCA). Further analyses for identifying metabolites underlying resistance or susceptibility were done using Partial Least Square - Discriminant Analyses (PLS-DA). In these supervised PLS-DA models, the resistance classification of each accession was included in the model. All plants were used in the metabolomics screening, and a subset was also used for the thrips bio-assays. For the set of plants of which we obtained both the metabolomics data as well as thrips damage estimates, we also performed Partial Least Square Regression analyses where \% damage data was regressed against the full metabolomic profiles. The PLS(-DA) models were cross-validated with permutation tests ( 999 permutations). $\mathrm{Q}^{2}$ values of all permuted models were compared to the $\mathrm{Q}^{2}$ values of the real data. $\mathrm{Q}^{2}$ values of the real data should be higher than 0.5 , and all permuted $\mathrm{Q}^{2}$ should be lower than the real $\mathrm{Q}^{2}$. Significance of the PLS models was also tested with cross-validated ANOVA (Eriksson et al. 2008; Triba et al. 2015). Subsequently, the PLS loading plots and variable influence on the projection (VIP) loadings of significant models were used to make an initial selection of clusters/ mass signal of interest (VIP > 2). To confirm our PLS(-DA) results, relative abundance of the clusters/mass signals from this initial cluster/mass selection were correlated with \% thrips damage at individual plant level (Spearman rank correlations). Those masses/clusters that showed a significant or near significant correlation with thrips damage, either positive or negative, were selected for metabolite annotation.

\section{Results}

\section{Thrips Feeding Preference}

The thrips leaf disc choice assay showed significant differences in feeding damage among the 11 accessions in both the vegetative stage and the generative stage (Fig. 1, Friedman ANOVA, vegetative stage $\chi^{2}=57.6, d f=10$, $P<0.0001$; generative stage $\chi^{2}=38.6, d f=10, P<0.0001$; see Table $\mathrm{S} 2$ for pairwise differences between the accessions). In our leaf disc assay, feeding was lowest on accession RU 63 and high on RU 43 in both the vegetative stage and generative stage (Fig. 1). For some accessions, thrips preference depended on plant developmental stage. For example, RU 34 received relatively little damage in the vegetative stage, but became more susceptible in the generative stage. Similarly, RU 52 became more susceptible when flowering. In contrast, RU 13 and RU 19 were susceptible in the 
Fig. 1 Mean percentage Frankliniella occidentalis leaf damage (+SE) per accession in choice tests with leaf discs of 11 Capsicum accessions a) when plants were 4 weeks old (vegetative) or b) flowering (generative). $\mathrm{X}$-axis labels indicate RU seedbank accession numbers (see Table 1). Accessions are ordered based on feeding preference in the vegetative stage. Overall differences between accessions are significant in both vegetative (A) and flowering (B) stage (both $\mathrm{P}<0.0001, P$ - values are based on Friedman ANOVAs for dependent data, for pairwise differences between accessions see Table S2). The letters $r$ and s indicate classification of the accession as either resistant ( $\mathrm{r}<9 \%$ damage) or susceptible (s $>9 \%$ damage). $N=8-10$ per accession
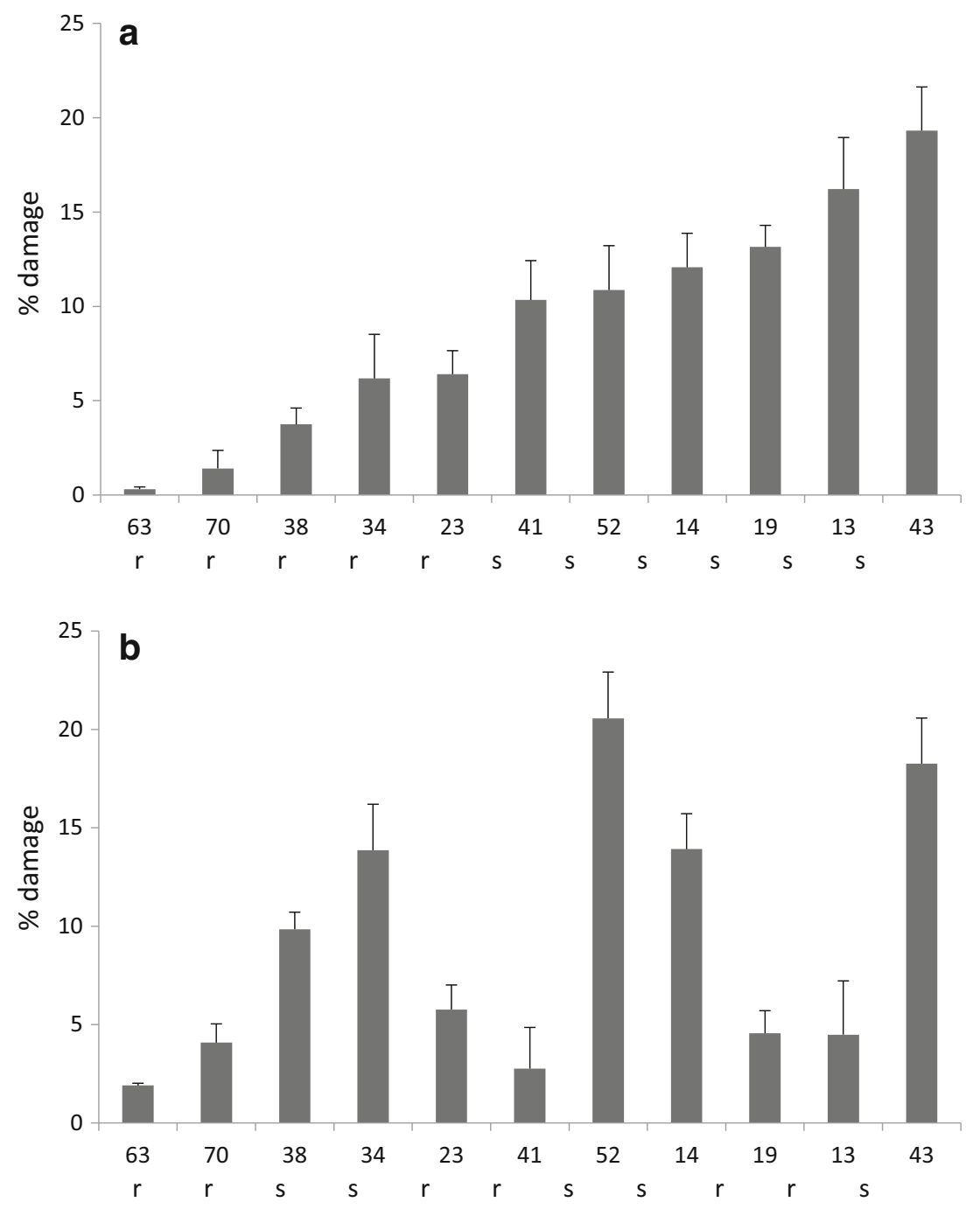

vegetative stage but became more resistant when the plants where flowering (Fig. 1).

\section{Metabolomic Profiling - Mass Signals Detected}

After data filtering, the negative ionization mode yielded 24,531 detected mass signals and the positive ionization mode 52,530 mass signals over all samples. The number of total detected masses in the negative mode differed significantly among accessions (ANOVA, $d f=10, F=$ 40.6, $P<0.001)$.

In some accessions there was a significant increase in number of detected masses in the generative stage compared to the vegetative stage (accession $\mathrm{x}$ developmental stage: $F=13.42, P<0.001$, Table 1 ). This was most pronounced in RU 13 (Table 1), which also was more resistant in the generative than in the vegetative stage (Fig. 1). In the generative stage, resistant accessions had on average significantly more detected masses than susceptible accessions (Table 1, ANOVA, $d f=1, F=11.36, P=$ $0.008)$. In the vegetative stage, there was no difference in number of detected masses between resistant and susceptible accessions (Table 1, ANOVA, $d f=1, F=1.24, P=$ 0.292) .

\section{Metabolomic Profiling - Multivariate Analyses}

The PCA of all data in both negative and positive ionization modes showed a clear separation of the metabolomics profiles based on species (PC 1) and developmental stage (PC 2) (Fig. 2, Fig. S1). Based on these results, further analyses in search for resistance factors in the metabolomics dataset were performed per species and developmental stage separately. Firstly, unsupervised PCA analyses of each species and developmental stage already showed a grouping based on resistance class (Fig. S2) and on accessions (Fig. S3). Subsequent supervised PLS-DAs which incorporated the resistance classification of each accession in the models, showed a clear separation of 
Table 1 Radboud University (RU) codes of Capsicum accessions, species, and the average number of detected mass peaks $( \pm \mathrm{SE})$ in leaves per accession at the vegetative or generative developmental stage in the negative ionization mode of the MS are indicated. $P$ values of Tukey post-hoc tests after ANOVA indicate differences between developmental stages.
Resistance column indicates whether an accession was classified as resistant (r) or susceptible (s) based on the choice assay results from Fig. 1. Note that this may change from the vegetative stage to the generative stage. $N=8-16$ per accession

\begin{tabular}{|c|c|c|c|c|c|c|}
\hline \multirow[b]{2}{*}{ RU no. } & \multirow[b]{2}{*}{ Species } & \multicolumn{4}{|c|}{ Detected mass peaks } & \multirow[b]{2}{*}{$P$} \\
\hline & & Vegetative & Resistance & Generative & Resistance & \\
\hline 63 & C. апnиum & $6561( \pm 87)$ & $\mathrm{r}$ & $7237( \pm 77)$ & $\mathrm{r}$ & $<0.0001$ \\
\hline 23 & C. апnиит & $6852( \pm 81)$ & $\mathrm{r}$ & $7099( \pm 66)$ & $\mathrm{r}$ & 0.065 \\
\hline 19 & C. аппиит & $6616( \pm 119)$ & $\mathrm{s}$ & $7171( \pm 75)$ & $\mathrm{r}$ & $<0.0001$ \\
\hline 34 & C. апnиит & $6507( \pm 109)$ & $\mathrm{r}$ & $5948( \pm 122)$ & $\mathrm{s}$ & $<0.0001$ \\
\hline 43 & C. аппиит & $6066( \pm 146)$ & $\mathrm{s}$ & $5943( \pm 134)$ & $\mathrm{s}$ & 0.363 \\
\hline 52 & C. аппиит & $5673( \pm 107)$ & $\mathrm{s}$ & $5697( \pm 196)$ & $\mathrm{s}$ & 0.241 \\
\hline 14 & C. аппиит & $6143( \pm 78)$ & $\mathrm{s}$ & $7052( \pm 132)$ & $\mathrm{s}$ & $<0.0001$ \\
\hline 38 & C. chinense & $5753( \pm 91)$ & $\mathrm{r}$ & $5939( \pm 65)$ & $\mathrm{s}$ & 0.194 \\
\hline 41 & C. chinense & $5591( \pm 44)$ & $\mathrm{s}$ & $6633( \pm 65)$ & $\mathrm{r}$ & $<0.0001$ \\
\hline 70 & C. chinense & $5746( \pm 96)$ & $\mathrm{r}$ & $6444( \pm 101)$ & $\mathrm{r}$ & $<0.0001$ \\
\hline 13 & C. chinense & $5771( \pm 55)$ & $\mathrm{s}$ & $7013( \pm 141)$ & $\mathrm{r}$ & $<0.0001$ \\
\hline \multirow[t]{2}{*}{ Average } & Resistant & $6341( \pm 67)$ & & $7012( \pm 50)$ & & \\
\hline & Susceptible & $6041( \pm 61)$ & & $6077( \pm 82)$ & & \\
\hline
\end{tabular}

metabolomic profiles based on resistance class (Fig. 3, Fig. S4). Cross-validation permutation tests of the PLS-DA models showed that all models were significant and not overfitting (CV-ANOVA all $P<0.001$; all $\mathrm{Q}^{2}>0.75$, all $\mathrm{Q}^{2}$ permuted models $<0.3$ ). For those plants of which we obtained both the metabolomics data as well as thrips damage estimates, we also linked thrips preference to metabolomic profiles by using PLS regression models. These PLS regression models of C. annuum were significant and showed a separation of metabolomics profiles based on thrips damage levels (Fig. 4, Fig. S5). For $C$. chinense these PLS regression models were not significant $\left(P>0.7, \mathrm{Q}^{2}<0.2\right)$, likely due to smaller sample size for this species, and were thus not used for further analyses.

\section{Metabolite Identification}

The PLS-DA loading plots and PLS regression loading plots were used to select clusters/masses that contributed most to the differences among groups and damage levels. Spearman correlations further showed (near) significant correlations between relative abundance of part of these masses and percentage thrips damage (Table 2). Annotation of these masses
Fig. 2 Principal Component Analyses (PCA) of LC-MS untargeted metabolomics analyses of Capsicum leaves in negative ionization mode. Different colors indicate different species and developmental stages. Ann veg: $C$. annuum vegetative stage (dark blue dots), Ann_gen:

C. annuum generative stage (green dots), Chin_veg: C. chinense vegetative stage (yellow dots), Chin_gen: C. chinense generative stage (red dots). Light blue dots indicate the pool samples

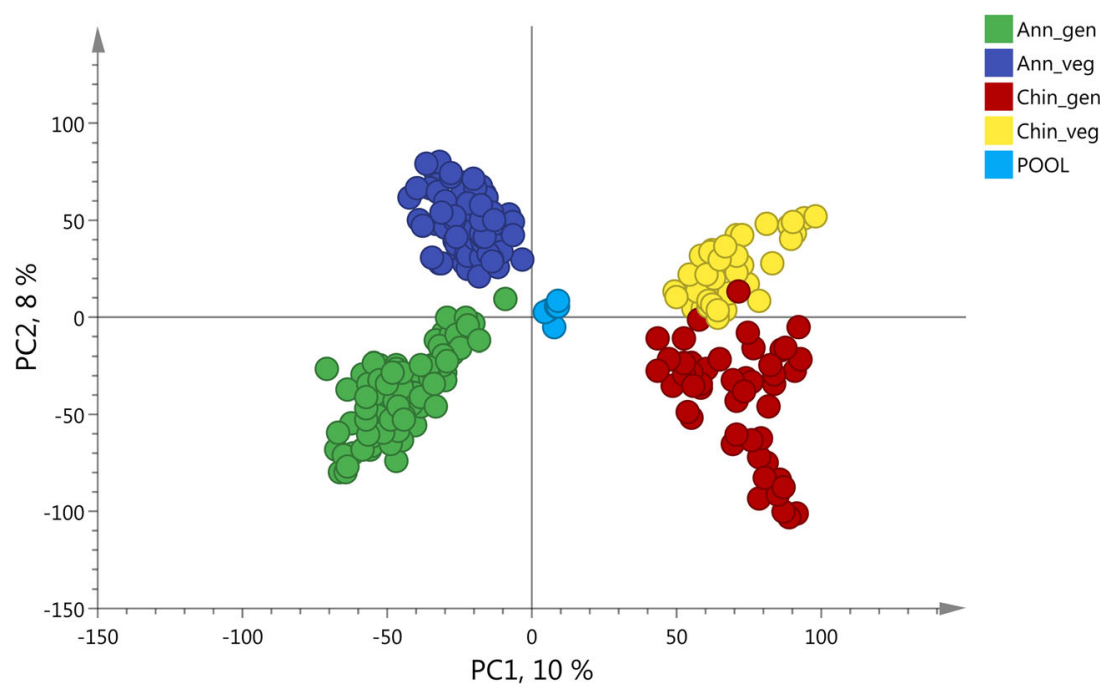


a C. annuum vegetative

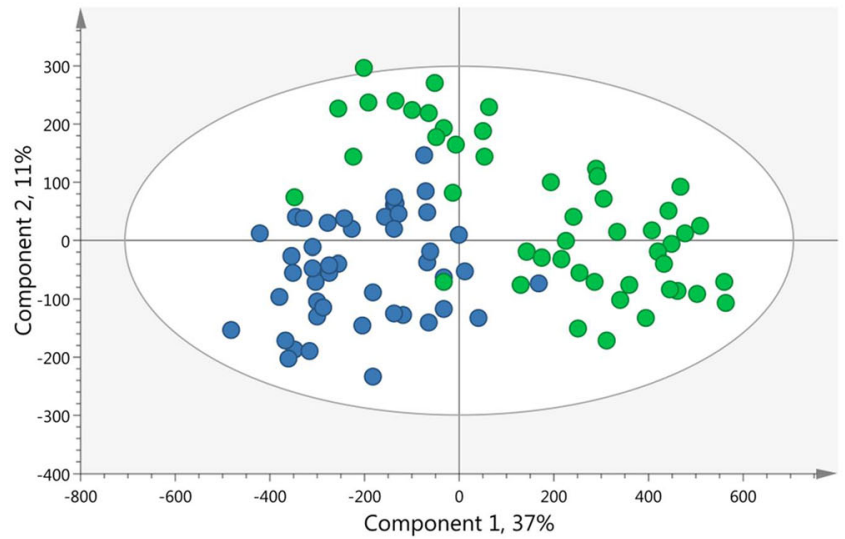

C C. annuum generative

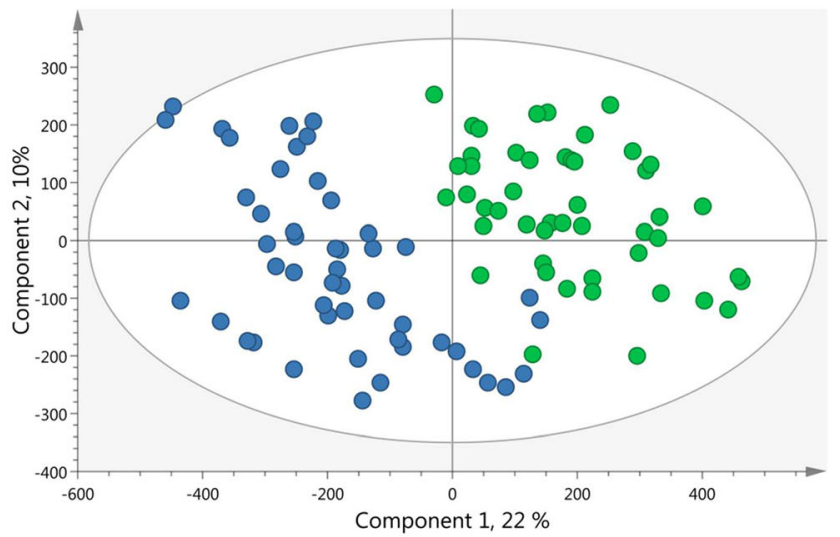

Fig. 3 Partial Least Square- Discriminant Analysis (PLS-DA) plots of LC-MS mass clusters (MIC) per Capsicum species and developmental stage. a) $C$. annuum vegetative stage, b) $C$. annuum generative stage, $\mathbf{c})$ $C$. chinense vegetative stage, d) $C$. chinense generative stage. Green dots

based on MS(MS) fragmentation patterns revealed that the metabolites that were related to resistance were mostly capsianosides, a group of acyclic diterpene glycosides (DTG) (Table 2). The relative abundance of both monomer capsianosides and dimer capsianosides were significantly negatively correlated with thrips preference (Table 2). While b C. chinense vegetative

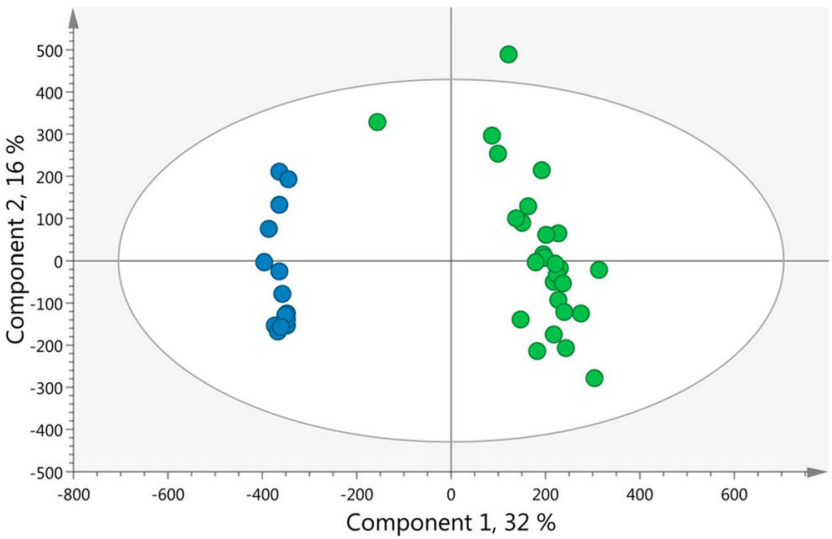

d C. chinense generative

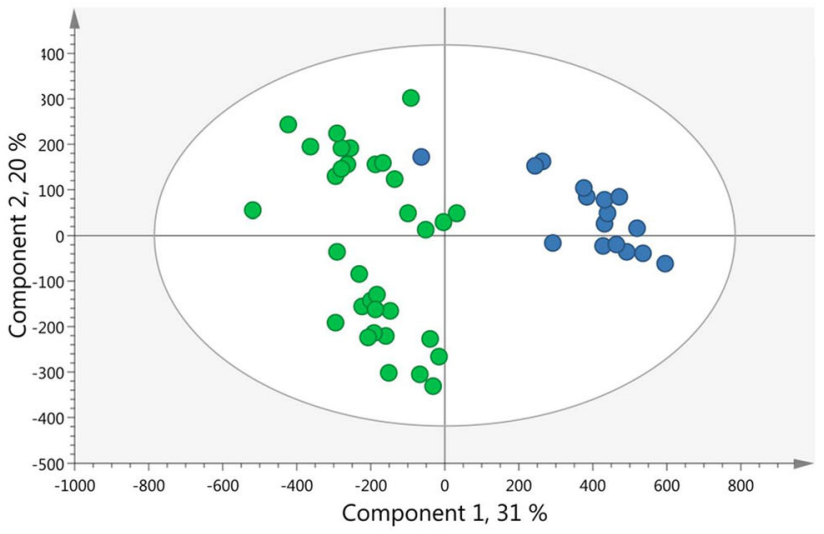

indicate plants of resistant accessions, blue dots indicate plants of susceptible accessions. All models were cross-validated with permutation tests $\left(\mathrm{Q}^{2}>0.75, P<0.0001\right) . N=8-16$ per accession

Capsianoside II was more abundant in C. annuиm compared to $C$. chinense, Capsisanoside VI was more abundant in C. chinese, indicating species-specific capsianoside profiles (Fig. 5ab, Fig. S6). Capsianoside VI emerged from the C. chinense PLS-DA as related to resistant accessions, but was nevertheless poorly directly correlated with thrips
Fig. 4 Partial Least Square regression plots of Capsicum annuum LC-MS metabolomic profiles (MIC) and \% thrips damage. a) plants in vegetative stage, b) generative stage. More blue colors indicate less thrips damage. Both models were significant $\left(\mathrm{Q}^{2}>0.6, P<0.005\right)$.

$N=6-10$ per accession
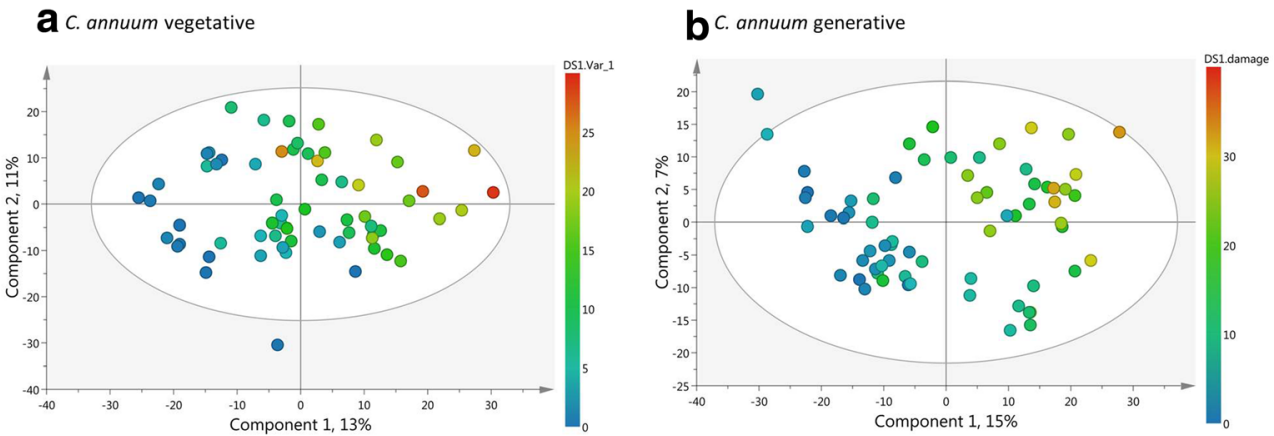
Table 2 Relevant metabolites in Capsicum spp. related thrips (Frankliniella occidentalis) resistance/susceptibility. Annotation based on MS(MS) fragmentation patterns in both positive and negative ionization mode. Annotation of capsianosides was based on presence of 271.24 aglycon fragment in positive ionization mode. $\mathrm{m} / \mathrm{z}$ : mass-tocharge ratio, Rt: retention time in minutes, $\mathrm{R}_{\mathrm{s}}$ : Spearman Rank
Correlation Coefficient of correlation between relative peak abundance and \% thrips damage per species/developmental stage. Small letters after $\mathrm{R}_{\mathrm{s}}$ indicate species/stage(s) in which a correlation(s) was found; ${ }^{\mathrm{av}}$ ) $C$. annuum vegetative, ${ }^{\mathrm{ag}}$ ) $C$. annuum generative stage, ${ }^{\mathrm{cv}}$ ) $C$. chinense vegetative stage, ${ }^{\mathrm{cg}}$ ) $C$. chinense generative stage. Lowest $\mathrm{R}_{\mathrm{s}}$ and $P$ value are given in case a metabolite was pinpointed in multiple analyses

\begin{tabular}{|c|c|c|c|c|c|}
\hline Compound no. & $m / z \mathrm{MH}-$ & Rt & Molecular formula & Annotation & $\mathrm{R}_{\mathrm{s}}$ \\
\hline 1 & 563.1407 & 14.9 & $\mathrm{C}_{26} \mathrm{H}_{28} \mathrm{O}_{14}$ & Apigenin-O-glycoside & $-0.35^{+\mathrm{cv}}$ \\
\hline 2 & 679.1543 & 17.3 & $\mathrm{C}_{30} \mathrm{H}_{32} \mathrm{O}_{18}$ & Luteolin methyl ether & $-0.37^{+\mathrm{cv}}$ \\
\hline 3 & 921.4693 & 23.6 & $\mathrm{C}_{44} \mathrm{H}_{74} \mathrm{O}_{20}$ & Capsianoside VI & $-0.17^{\mathrm{cg}}$ \\
\hline 4 & 1083.5238 & 21.7 & $\mathrm{C}_{50} \mathrm{H}_{84} \mathrm{O}_{25}$ & Capsianoside II & $-0.39 * *^{\mathrm{av}, \mathrm{ag}}$ \\
\hline 5 & 1169.5247 & 22.5 & $\mathrm{C}_{53} \mathrm{H}_{86} \mathrm{O}_{28}$ & Monomer Capsianoside & $-0.52 * * *$ av,ag \\
\hline 6 & 1169.5248 & 23.0 & $\mathrm{C}_{53} \mathrm{H}_{86} \mathrm{O}_{28}$ & Monomer Capsianoside & $-0.50 * * *$ av \\
\hline 7 & 1185.5165 & 20.9 & $\mathrm{C}_{53} \mathrm{H}_{86} \mathrm{O}_{29}$ & Capsianoside II + malonyl & $-0.50 * * * \mathrm{ag}$ \\
\hline 8 & 1735.7971 & 27.5 & $\mathrm{C}_{82} \mathrm{H}_{128} \mathrm{O}_{39}$ & Dimer Capsianoside & $-0.72 * * * \mathrm{ag}$ \\
\hline 9 & 1563.7969 & 28.0 & $\mathrm{C}_{76} \mathrm{H}_{124} \mathrm{O}_{33}$ & Dimer Capsianoside & $-0.39 * \mathrm{av}, \mathrm{ag}$ \\
\hline 10 & 1649.7977 & 28.2 & $\mathrm{C}_{79} \mathrm{H}_{126} \mathrm{O}_{36}$ & Dimer Capsianoside & $-0.70 * * *$ av,ag \\
\hline 11 & 1735.7976 & 28.7 & $\mathrm{C}_{82} \mathrm{H}_{128} \mathrm{O}_{39}$ & Dimer Capsianoside & $-0.77 * * * \mathrm{ag}$ \\
\hline 12 & 1735.7971 & 29.5 & $\mathrm{C}_{82} \mathrm{H}_{128} \mathrm{O}_{39}$ & Dimer Capsianoside & $-0.76^{* * * a v, a g}$ \\
\hline 13 & 341.1089 & 2.1 & $\mathrm{C}_{12} \mathrm{H}_{22} \mathrm{O}_{11}$ & Sucrose & $0.50 * * * a \mathrm{~g}$ \\
\hline 14 & 343.1074 & 21.0 & $?$ & Unknown & $0.70^{* * *} *^{\mathrm{cv}}$ \\
\hline 15 & 353.0876 & 8.7 & $\mathrm{C}_{16} \mathrm{H}_{18} \mathrm{O}_{9}$ & Chlorogenic acid & $0.46^{* * * a g}$ \\
\hline 16 & 371.2065 & 14.3 & $\mathrm{C}_{13} \mathrm{H}_{22} \mathrm{O}_{2}$ hexoside & Unknown & $0.40 * *$ av,ag \\
\hline 17 & 473.1093 & 17.7 & $\mathrm{C}_{24} \mathrm{H}_{21} \mathrm{O}_{13}$ & Apigenin-O-malonyl hexoside & $0.60 * * * a \mathrm{ag}$ \\
\hline 18 & 533.0937 & 15.9 & $\mathrm{C}_{24} \mathrm{H}_{22} \mathrm{O}_{14}$ & Luteolin-O-malonyl hexoside & $0.50 * * * a g, c v$ \\
\hline 19 & 581.1529 & 9.2 & $\mathrm{C}_{26} \mathrm{H}_{30} \mathrm{O}_{15}$ & Naringenin-C-hexoside-pentoside & $0.56^{* * * a v}$ \\
\hline 20 & 649.1407 & 16.9 & $\mathrm{C}_{29} \mathrm{H}_{30} \mathrm{O}_{17}$ & Malonylapiin & $0.45 * * *$ av,ag \\
\hline 21 & 665.1389 & 15.4 & $\mathrm{C}_{29} \mathrm{H}_{30} \mathrm{O}_{18}$ & Luteolin-diglucoside' malonate & $0.83 * * * \mathrm{cv}, \mathrm{av}$ \\
\hline
\end{tabular}

$+P<0.10$

$* P \leq 0.01$

$* * P \leq 0.001$

$* * * P \leq 0.0001$

preference (Table 2). The dimer capsianosides related to thrips resistance were mostly only detected here in $C$. аппиит (Fig. 5c, Fig. S6, Fig. S7). Furthermore, two flavone glycosides were related to resistance (compound 1 and 2), although other flavone glycosides (compounds 18-22) were related to susceptibility (Table 2). These flavone glycosides related to thrips susceptibility mostly had a malonyl group (compound 17-18 and 19-20), such as malonylapiin (Table 2). Chlorogenic acid and sucrose were also positively correlated with thrips damage (Table 2). The relative abundance of sucrose and in particular chlorogenic acid was high in leaves of thrips susceptible accession RU 43 when plants were flowering (Fig. 5de). Furthermore, sucrose levels in RU 13 dropped in the flowering stage, while they increase in RU 52 and RU 34 (Fig. 5d). This pattern matches with RU 13 becoming more resistant and RU 52 and RU 34 becoming more susceptible in the flowering stage compared to the vegetative stage (Fig. 1).

\section{Discussion}

Our untargeted metabolomics analyses in combination with thrips feeding assays of multiple Capsicum accessions revealed metabolites that were related to thrips resistance and susceptibility. Several monomer and dimer acyclic diterpene glycosides (DTG) were related to resistance, while sucrose, flavone glycosides and chlorogenic acid were related to susceptibility. To our knowledge, this is the first study that links the dimer DTG to thrips resistance. Our results are in line with other recent studies on DTG and highlight the emerging role of DTG as defenses against insects in Solanaceae (Heiling et al. 2010; Poreddy et al. 2015; Maharijaya et al. 2018).

The thrips choice assays showed significant differences in thrips feeding among the selected Capsicum accessions. Within the selected accessions, RU 63 was the most resistant, which is consistent with earlier studies (Maharijaya et al. 2011) that included this and other accessions. RU 43 and 

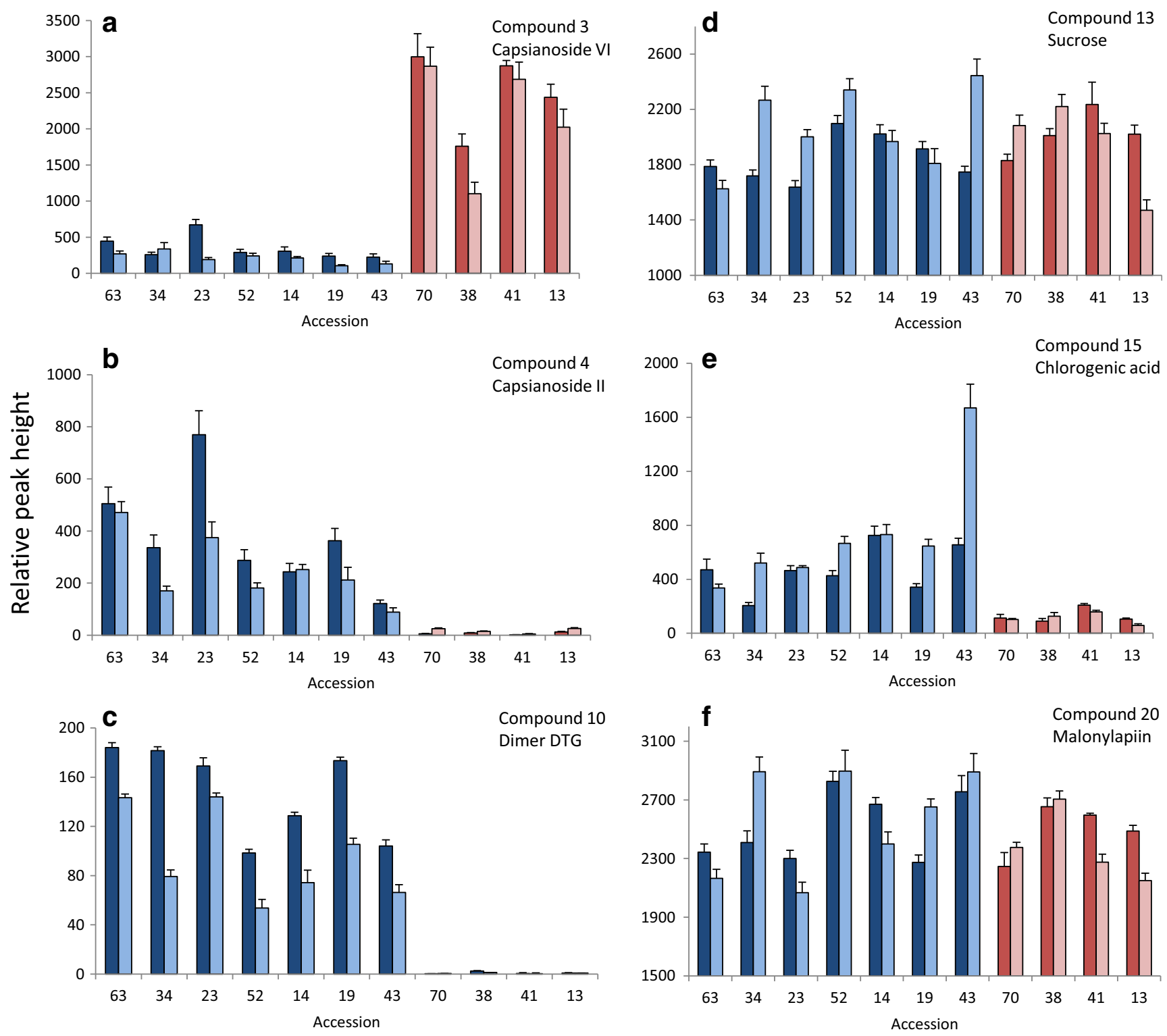

Fig. 5 Examples of relative abundance of peak heights of selected metabolites per Capsicum accession (+SE) in the vegetative stage (dark bars) and the flowering stage (light bars). C. апnиum accessions are indicated in blue, $C$. chinense accessions in red. Compound numbers

correspond with those in Table 2. a) 3. Capsianoside VI, b) 4 . Capsianoside II, c) 11. Dimer Capsianoside, d) 13. Sucrose, e) 15. Chlorogenic acid, f) 20. Malonylapiin. See Fig. S6 for relative abundances of all metabolites from Table 2

RU 52 were the most susceptible. Interestingly, three accessions became more resistant when they started flowering, while two other accessions became more susceptible. Agerelated resistance has been shown in disease resistance (e.g. Kus et al. 2002) and has also been described for resistance to insects (e.g. Beck 1964; Cipollini and Redman 1999; Stout et al. 2013). Generally, it is thought that changes in intrinsic factors, such as resource acquisition and allocation, as well as changes in extrinsic factors, such as herbivore selection pressures, drive the evolution of age-related resistance (Barton and Boege 2017). The chemical mechanisms behind age-related resistance of our Capsicum accessions remain unclear, although our data suggest this could be related to shifts in relative abundance of sucrose in the leaves.

C. annuum and C. chinense had species-specific metabolome profiles. Nevertheless, within each species and developmental stage, there were overall differences in metabolomic profiles between thrips resistant and susceptible Capsicum accessions. Metabolites that were negatively correlated with thrips damage were annotated as acyclic diterpene glycosides (DTG), so-called capsianosides in Capsicum species. There are two types of DTG in Capsicum; monomer capsianosides I-XVIII with either 17hydroxygeranyllinalool (HGL), 13-hydroxygeranyllinalool-16oic acid aglycone or geranyllinalool-16-oic acid as aglycone, 
and dimer capsianosides A-L, with HGL and 13hydroxygeranyllinalool-16-oic acid or geranyllinalool-16-oic acid as aglycones (Izumitani et al. 1990; Yahara et al. 1991; Lee et al. 2008). A recent mQTL (metabolite QTL) study linked four monomer capsianosides from Capsicum with thrips survival (Maharijaya et al. 2018). Our metabolomics analyses revealed that also dimer capsianosides are related to thrips resistance. We detected ten capsianosides that were related to thrips resistance, five of which were dimers. Interestingly, of these ten capsianosides only compound \#7 (Capsianoside II + malonyl) was also found in the mQTL study of Maharijaya et al. (2018). Capsianosides may also have antimicrobial activity (Bacon et al. 2017). Similar monomer 17-hydroxygeranyllinalool diterpene glycosides (HGL-DTG) in Nicotiana species are acting as direct defenses against multiple insect herbivores (Snook et al. 1997; Heiling et al. 2010). The 17-hydroxygeranyllinalool diterpene backbone is thought to be the active component of the DTG (Snook et al. 1997). The sugars and malonyl groups that are attached to this terpene backbone are proposed to enable transport and storage inside the plant and prevent autotoxicity (Heiling et al. 2010).

Our data suggest species-specific abundance of particular capsianosides. For example, capsianoside VI was more abundant in C. chinense than in C. annuum, and capsianoside II was more abundant in $C$. annuum. Differential activity of modifying enzymes such as glycosyltransferases may lead to differences in capsianosides profiles among Capsicum accessions and species (Wahyuni et al. 2014), as was shown in Nicotiana (Heiling et al. 2016). A major QTL for thrips resistance co-localized with two DTG (Maharijaya et al. 2018). The capsianosides are also present in Capsicum fruits (Wahyuni et al. 2013), and it is unclear how high levels may affect taste or quality of the fruits, although they may have dietary benefit (De Marino et al. 2006). Interestingly, in fruits the mQTLs for capsianosides were localized at different chromosomes than the mQTLs of some capsianosides present in the leaves (Maharijaya et al. 2018; Wahyuni et al. 2014). We studied the constitutive chemical defenses of Capsicum, but it seems likely that the capsianosides also play a role in herbivore induced defenses. DTG synthesis in N. attenuata is induced upon herbivore feeding, which is regulated by the jasmonate signaling pathway (Heiling et al. 2010). Elucidating the molecular pathways and genes involved in capsianoside production, induction and diversity may shed light on the genetic mechanisms of DTGbased insect resistance in Capsicum.

At least 18 monomer and 12 dimer capsianosides have been described thus far in Capsicum species (Lee et al. 2008; Yahara et al. 1991,). In our additional MS/MS analysis of selected samples of $C$. annuum RU 63 we detected 6 monomer and 12 dimer capsianosides (Fig. S7). Not all of these emerged from our untargeted metabolomics study as linked to thrips resistance. It is possible that not all capsianosides are equally effective against $F$. occidentalis. Bioactivity of DTG may depend on their glycosylation pattern (Poreddy et al. 2015), but this needs further research with targeted analyses specifically aimed at detecting both monomer and dimer capsianosides. Furthermore, due to limitations of our MS-based analytical platform, mainly the inability to assign exact sugar positions and linkages to these complex molecules (Heiling et al. 2016), detailed annotation of the dimer capsianosides was not possible.

Several flavone glycosides (compound 17-21) were positively correlated with thrips damage, although two other flavones were correlated with resistance in $C$. chinense (compound 1-2). Structurally related metabolites can differ in their effects on insects. For the flavones for example, luteolin-D-glycoside from C. annuum was not deterrent to the leaf miner Lyriomyza trifolli, while the structurally related luteolin-apiosyl-glucoside was highly deterrent (Kashiwagi et al. 2005). Luteolin itself was toxic to thrips (Leiss et al. 2013). Here, we found that an apigenin-Oglycoside and a luteolin methyl ether were related to thrips resistance, but that some malonylated luteolin and apigenin glycosides were related to susceptibility to thrips. The role of malonylation in defense needs further study (Heiling et al. 2010).

Sucrose was related to thrips susceptibility, which is not surprising because thrips need the sugars from the plant to survive and grow (Nielsen et al. 2010). Chlorogenic acid was also related to susceptibility but has been related to insect resistance in other plant species (e.g. Leiss et al. 2009; Dillon et al. 2017). In our study, chlorogenic acid was particularly abundant in one of our most susceptible $C$. annuum accessions in the flowering stage (RU 43). Our results showed that chlorogenic acid per se is not an effective defense against thrips in Capsicum. Possibly, differences in polyphenol oxidase activity (enzymes involved in oxidative activation of phenolic compounds) among species determine the role of chlorogenic acid in plant - insect interactions (Appel 1993). Moreover, the effectivity of plant compounds is likely determined by the background metabolome in which they are present, which is different for each species and genotype. In a similar way, gene function may alter depending on the background genome (Chandler et al. 2013). Artificial diet experiments with alkaloids and chlorogenic acid showed that indeed the chemical background influenced the bioactivity of the combination of these compounds on thrips survival (Liu et al. 2018).

In a similar way, interactions between capsianosides and other compounds of the metabolomes of different accessions may alter the effect of capsianosides on resistance. Maharijaya et al. (2018) also suggest that several metabolites acting in concert may be responsible for thrips resistance in Capsicum. In our study, there was no clear trend that capsianoside abundance went up in those accessions that became more resistant in the flowering stage. Possibly, it is the combination of capsianosides and sucrose or other compounds that are important for resistance (e.g. high levels of capsianosides, low levels of sucrose). Metabolite richness or diversity of the metabolome in itself may further play a role in insect resistance (Macel et al. 2014). In our Capsicum study, resistant accessions had on average significantly higher 
chemical diversity than susceptible accessions in the flowering stage, although this was not significant in the vegetative stage. Compounds can act synergistically and a wider range of metabolites is thought to act as defenses against a wider range of attackers (Berenbaum et al. 1991).

Untargeted metabolomics provides a more comprehensive insight into the plant metabolome and can be used as a tool to screen for the unknowns that are important in plant - insect interactions. Furthermore, interactions between compounds cannot be revealed with targeted chemical analytical approaches. Once interesting metabolites have been identified, fine-tuned targeted chemical analytical procedures and additional experiments with gene-silenced plants and/or bioassays with pure compounds could further elucidate their effect on insects. The group of acyclic diterpene glycosides that we pinpointed in our pepper study have previously been related to broad spectrum insect resistance in Nicotiana species. Within the Solanaceae, DTG have been detected in the genus Capsicum spp., Lycium barbarum and several Nicotiana species, but were absent from Tomato (Solanum lycopersicum) and Potato (Solanum tuberosum) (Heiling et al. 2016). Similar DTG were found in Asteraceae (Akter et al. 2016). These are all fairly recent studies; it is thus possible that DTG occur in other plant species belonging to different plant families. Increasing the levels of DTG in elite breeding lines could be a way to increase insect resistance in pepper crops, provided that there is no trade-off with yield or food quality. Using natural variation of insect resistance in wild relatives of crops in combination with novel chemical and genetic technologies can lead to the discovery of new sources of insect resistance that may enhance crop protection.

Acknowledgements We thank Bert Schipper (Wageningen Research Bioscience), Francel Verstappen (Wageningen University) and Rick Hoogeveld (Radboud University) for their technical assistance, Young Choi and Peter Klinkhamer (Institute of Biology Leiden) for the kind use of their facilities, and the Radboud greenhouse staff for their assistance. Nicole M. van Dam gratefully acknowledges the support of the German Centre for Integrative Biodiversity Research (iDiv) Halle-Jena-Leipzig funded by the German Research Foundation (FZT 118). This research was funded by the NWO Domain Applied and Engineering Sciences (AES) and is part of the Green defense Against Pest (GAP) program, project 13552.

Open Access This article is distributed under the terms of the Creative Commons Attribution 4.0 International License (http:// creativecommons.org/licenses/by/4.0/), which permits unrestricted use, distribution, and reproduction in any medium, provided you give appropriate credit to the original author(s) and the source, provide a link to the Creative Commons license, and indicate if changes were made.

\section{References}

Akter R, Uddin SJ, Tiralongo J, Grice ID, Tiralongo E (2016) A new cytotoxic diterpenoid glycoside from the leaves of Blumea lacera and its effects on apoptosis and cell cycle. Nat Prod Res 30:2688-2693
Appel HM (1993) Phenolics in ecological interactions: the importance of oxidation. J Chem Ecol 19:1521-1552

Bacon K, Boyer R, Denbow C, O’Keefe S, Neilson A, Williams R (2017) Antibacterial activity of jalapeño pepper (Capsicum annuum var. annuиm) extract fractions against select foodborne pathogens. Food Sci Nutr 5:730-738

Barton KE, Boege K (2017) Future directions in the ontogeny of plant defence: understanding the evolutionary causes and consequences. Ecol Lett 20:403-411

Barton KE, Koricheva J (2010) The ontogeny of plant defense and herbivory: characterizing general patterns using meta-analysis. Am Nat 175:481-493

Bass C, Jones CM (2018) Pests and resistance: resistance to pesticides in arthropod crop pests and disease vectors: mechanisms, models and tools. Curr Opin Insect Sci 27:iv-vii

Beck SD (1964) Resistance of plants to insects. Annu Rev Entomol 10: 207-232

Berenbaum MR, Nitao JK, Zangerl AR (1991) Adaptive significance of furanocourmarin diversity in Pastinaca sativa (Apiaceae). J Chem Ecol 17:207-215

Bielza P (2008) Insecticide resistance management strategies against the western flower thrips, Frankliniella occidentalis. Pest Manag Sci 64:1131-1138

Chandler CH, Chari S, Dworkin I (2013) Does your gene need a background check? How genetic background influences the analysis of mutations, genes and evolution. Trends Genet 29:358-366

Cipollini DF, Redman AM (1999) Age-dependent effects of jasmonic acid treatment and wind exposure on foliar oxidase activity and insect resistance in tomato. J Chem Ecol 25:271-281

De Marino S, Borbone N, Gala F, Zollo F, Fico G, Pagiotti R, Iorizzi M (2006) New constituents of sweet Capsicum annuum L. fruits and evaluation of their biological activity. J Agric Food Chem 54:7508-7516

De Vos RCH, Moco S, Lommen A, Keurentjes JJB, Bino RJ, Hall RD (2007) Untargeted large-scale plant metabolomics using liquid chromatography coupled to mass spectrometry. Nat Protoc 2(4):778-791

Dillon FM, Chludil HD, Zavala JA (2017) Solar UV-B radiation modulates chemical defenses against Anticarsia gemmatalis larvae in leaves of field-grown soybean. Phytochemistry 141:27-36

Eriksson L, Trygg J, Wold S (2008) CV-ANOVA for significance testing of PLS and OPLS $®$ models. J Chemom 22:594-600

Fery JM, Schalk RL (1991) Resistance in pepper (Capsicum annuum L.) to western flower thrips (Frankliniella occidentalis (Pergande)). HortScience 26:1073-1074

Gols R, Bukovinszky T, Van Dam NM, Dicke M, Bullock JM, Harvey JA (2008) Performance of generalist and specialist herbivores and their endoparasitoids differs on cultivated and wild Brassica populations. J Chem Ecol 34:132-143

Hallmann CA, Foppen RPB, Van Turnhout CAM, De Kroon H, Jongejans E (2014) Declines in insectivorous birds are associated with high neonicotinoid concentrations. Nature 511:341-343

Heiling S, Schuman MC, Schoettner M, Mukerjee P, Berger B, Schneider B, Jassbi AR, Baldwin IT (2010) Jasmonate and ppHsystemin regulate key malonylation steps in the biosynthesis of 17 hydroxygeranyllinalool diterpene glycosides, an abundant and effective direct defense against herbivores in Nicotiana attenuata. Plant Cell 22:273-292

Heiling S, Khanal S, Barsch A, Zurek G, Baldwin IT, Gaquerel E (2016) Using the knowns to discover the unknowns : MS-based dereplication uncovers structural diversity in 17-hydroxygeranyllinalool diterpene glycoside production in the Solanaceae. Plant J 85:561-577

Izumitani Y, Yahara S, Nohara T (1990) Novel aclycic diterpene glycosides, Capsianosides A-F and I-V from Capsicum plants (Solanaceous Studies . XVI). Chem Pharm Bull 28:1299-1307

Kashiwagi T, Horibata Y, Mekuria DB, Tebayashi S, Kim C (2005) Ovipositional deterrent in the sweet pepper, Capsicum annuum, at 
the mature stage against Liriomyza trifolii (Burgess). Biosci Biotechnol Biochem 69:1831-1835

Kim TJ, Choi J, Kim KW, Ahn SK, Ha SH, Choi Y, Park NI, Kim JK (2017) Metabolite profiling of peppers of various colors reveals relationships between tocopherol, carotenoid, and phytosterol content. J Food Sci 82:2885-2893

Kirk WDJ, Terry LI (2003) The spread of the western flower thrips Frankliniella occidentalis (Pergande). Agric For Entomol 5:301-310

Kus JV, Zaton K, Sarkar R, Cameron RK (2002) Age-related resistance in Arabidopsis is a developmentally regulated defense response to Pseudomonas syringae. Plant Cell 14:479-490

Lee J, Kiyota N, Ikeda T, Nohara T (2008) Three new acyclic diterpene glycosides from aerial parts of paprika and pimiento. Chem Pharm Bull 56:582-584

Lee HA, Kim S, Kim S, Choi D (2017) Expansion of sesquiterpene biosynthetic gene clusters in pepper confers nonhost resistance to the Irish potato famine pathogen. New Phytol 215:1132-1143

Leiss KA, Maltese F, Choi YH, Verpoorte R, Klinkhamer PGL (2009) Identification of chlorogenic acid as a resistance factor for thrips in chrysanthemum. Plant Physiol 150:1567-1575

Leiss KA, Cristofori G, Van Steenis R, Verpoorte R, Klinkhamer PGL (2013) An eco-metabolomic study of host plant resistance to Western flower thrips in cultivated, biofortified and wild carrots. Phytochemistry 93:63-70

Liu X, Vrieling K, Klinkhamer PGL (2018) Phytochemical background mediates effects of pyrrolizidne alkaloids on western flower thrips. $\mathrm{J}$ Chem Ecol 45:116-127. https://doi.org/10.1007/s10886-018-1009-2

Lommen A (2009) MetAlign: Interface-driven, versatile metabolomics tool for hyphenated full-scan mass spectrometry data preprocessing. Anal Chem 81:3079-3086

Macel M, van Dam NM, Keurentjes JJB (2010) Metabolomics: the chemistry between ecology and genetics. Mol Ecol Resour 10:583-593

Macel M, de Vos RCH, Jansen JJ, van der Putten WH, van Dam NM (2014) Novel chemistry of invasive plants: exotic species have more unique metabolomic profiles than native congeners. Ecol Evol 4:2777-2786

Maharijaya A, Vosman B, Steenhuis-Broers G, Harpenas A, Purwito A, Visser RGF, Voorrips RE (2011) Screening of pepper accessions for resistance against two thrips species (Frankliniella occidentalis and Thrips parvispinus). Euphytica 177:401-410

Maharijaya A, Vosman B, Verstappen F, Steenhuis-Broers G, Mumm R, Purwito A, Visser RGF, Voorrips RE (2012) Resistance factors in pepper inhibit larval development of thrips (Frankliniella occidentalis). Ent Exp et Appl 145:62-71

Maharijaya A, Vosman B, Pelgrom K, Wahyuni Y, de Vos RCH, Voorrips RE (2018) Genetic variation in phytochemicals in leaves of pepper (Capsicum) in relation to thrips resistance. Arthropod Plant Interact 0:1-9

Martín A, Hernández A, Aranda E, Casquete R, Velázquez R, Bartolomé T, Córdoba MG (2017) Impact of volatile composition on the sensorial attributes of dried paprikas. Food Res Int 100:691-697

Mokochinski JB, Mazzafera P, Sawaya ACHF, Mumm R, de Vos RCH, Hall RD (2018) Metabolic responses of Eucalyptus species to different temperature regimes. J Integr Plant Biol 60:397-411

Mollema C, Cole RA (1996) Low aromatic amino acid concentrations in leaf proteins determine resistance to Frankliniella occidentalis in four vegetable crops. Ent Exp et Appl 78:325-333

Nielsen M-C, Teulon DAJ, Chapman RB, Butler RC, Drayton GM, Philipsen H (2010) Comparison of life history parameters of two Frankliniella occidentalis (Thysanoptera: Thripidae) strains in New Zealand. Environ Entomol 39:303-311

Peters K, Worrich A, Weinhold A, Alka O, Balcke G, Birkemeyer C, Bruelheide H, Calf OW, Dietz S, Dührkop K et al (2018) Current challenges in plant eco-metabolomics. Int J Mol Sci 19:1-38

Poreddy S, Mitra S, Schöttner M, Chandran J, Schneider B, Baldwin IT, Kumar P, Pandit SS (2015) Detoxification of hostplant's chemical defence rather than its anti-predator co-option drives $\beta$-glucosidase- mediated lepidopteran counteradaptation. Nat Commun 6:8525. https://doi.org/10.1038/ncomms9525

Schoonhoven LM, Van Loon JJ, Dicke M (2005) Insect-plant biology. Oxford University Press, Oxford

Schweiger R, Baier MC, Persicke M, Müller C (2014) High specificity in plant leaf metabolic responses to arbuscular mycorrhiza. Nat Commun 5:3886

Shivaramu S, Jayanthi PDK, Kempraj V, Anjinappa R, Nandagopal B, Chakravarty AK (2017) What signals do herbivore-induced plant volatiles provide conspecific herbivores? Arthropod Plant Interact $11: 815-823$

Snook ME, Johnson AW, Severson RF, Teng Q, White RA Jr, Sisson VA, Jackson DM (1997) Hydroxygeranyllinalool glycosides from tobacco exhibit antibiosis activity in the tobacco budworm (Heliothis virescens (F.)). J Agric Food Chem 45:2299-2308

Steenbergen M, Abd-El-Haliem A, Bleeker P, Dicke M, Escobar-Bravo R, Cheng G, Haring MA, Kant MR, Kappers I, Klinkhamer PGL et al (2018) Thrips advisor: exploiting thrips-induced defences to combat pests on crops. J Exp Bot 69:1837-1848

Stout MJ, Hamm JC, Abbe I, Bergeron C (2013) The influence of rice plant age on susceptibility to the rice water weevil, Lissorhoptrus oryzophilus. J Appl Entomol 137:241-248

Tewksbury JJ, Reagan KM, Machnicki NJ, Carlo TA, Haak DC, Calderon Penaloza AL, Levey DJ (2008) Evolutionary ecology of pungency in wild chilies. Proc Natl Acad Sci 105:11808-11811

Tikunov YM, Laptenok S, Hall RD, Bovy A, de Vos RCH (2012) MSClust : a tool for unsupervised mass spectra extraction of chromatography-mass spectrometry ion-wise aligned data. Metabolomics 8:714-718

Triba MN, Le Moyec L, Amathieu R, Goossens C, Bouchemal N, Nahon P, Rutledge DN, Savarin P (2015) PLS/OPLS models in metabolomics: the impact of permutation of dataset rows on the K-fold crossvalidation quality parameters. Mol BioSyst 11:13-19

van der Hooft JJJ, Vervoort J, Bino RJ, de Vos RCH (2012) Spectral trees as a robust annotation tool in LC-MS based metabolomics. Metabolomics 8:691-703

Visschers IGS, van Dam NM, Peters JL (2018a) An objective highthroughput screening method for thrips damage quantitation using Ilastik and ImageJ. Ent Exp et Appl 166:508-515

Visschers IGS, van Dam NM, Peters JL (2018b) Quantification of thrips damage using Ilastik and ImageJ Fiji. Bio-protocol 8(8):e2806. https://doi.org/10.21769/BioProtoc.2806

Visschers IGS, Peters JL, van de Vondervoort JAH, Hoogveld RHM, van Dam NM (2019) Thrips resistance screening is coming of age: leaf position and ontogeny are important determinants of leaf-based resistance in pepper. Front Plant Sci 10 https://doi.org/10.3389/fpls. 2019.00510

Wahyuni Y, Ballester A, Tikunow Y, de Vos RCH, Pelgrom KTB, Maharijaya A, Sudarmonowati E, Bino RJ, Bovy AG (2013) Metabolomics and molecular marker analysis to explore pepper (Capsicum sp.) biodiversity. Metabolomics 9:130-144

Wahyuni Y, Stahl-Hermes V, Ballester A, de Vos RCH, Voorrips RE, Maharijaya A, Molthoff J, Zamora M, Sudarmonowati E, Maisonnave Arisi AC et al (2014) Genetic mapping of semi-polar metabolites in pepper fruits (Capsicum sp.): towards unravelling the molecular regulation of flavonoid quantitative trait loci. Mol Biol Evol 33:503-518

Weckwerth W (2003) Metabolomics in systems biology. Annu Rev Plant Biol 54:669-689

Yahara S, Kobayashi N, Izumitani Y, Nohara T (1991) New acyclic diterpene glycosides, capsianosides VI, G and $\mathrm{H}$ from the leaves and stems of Capsicum annuum L. Chem Pharm Bull 39:1358-3260

Yang JW, Yi HS, Kim H, Lee B, Lee S, Ghim SY, Ryu CM (2011) Whitefly infestation of pepper plants elicits defence responses against bacterial pathogens in leaves and roots and changes the below-ground microflora. J Ecol 99:46-56 